\title{
Glosse
}

\section{TAVI für alle!}

_ Es steht außer Frage: Die Entwicklung der kathetergestützten Aortenklappenimplantation (TAVI) ist eine der größten Fortschritte in der Kardiologie, um nicht zu sagen der Medizin insgesamt. Zunächst einmal war man ja etwas vorsichtig und hat diese neuen "Dinger" nur bei Patienten eingepflanzt, die sogar der Chirurg für nicht mehr operabel hielt - und das heißt ja schon etwas. Und als das dann ganz gut klappte, ging es Schritt für Schritt weiter.

Als nächstes wurden Patienten mit einem hohen, dann mit einem mittleren Risiko dieser segensreichen Intervention zugeführt, allerdings zum Verdruss der Herzchirurgen. Letzteres ist auch verständlich. Denn wer lässt sich schon gerne die Butter vom Brot nehmen. Und jetzt soll die Operation sogar ganz abgeschafft werden. Denn jeder Patient mit einer Aortenklappenstenose ist nach neuesten Studienergebnissen mit der Katheterintervention besser bedient als mit dem chirurgischen Klappenersatz. Kein Wunder, dass die Chirurgen diesen Siegeszug der Kardiologen als Feldzug gegen ihre Profession empfinden.
Aber das „Kardiologen-Märchen“ vom interventionellen Klappenersatz ist damit noch lange nicht zu Ende. Schon fragen die ersten Experten, warum man eigentlich warten soll, bis sich die Aortenstenose entwickelt hat. Wäre es nicht sinnvoller, bei jedem quasi als Geschenk zum 50. oder 60. Geburtstag eine TAVI einbauen zu lassen? Denn eine gesunde Klappe verspricht ja bekanntlich nichts Gutes, sie kann ja nur enger werden. Und dann wird alles sehr viel komplizierter.

Dazu kommt, dass es um die Finanzen in unserem Gesundheitssystem schon auf kürzere Sicht bekanntermaBen nicht gut bestellt ist. Man muss befürchten, dass solche teuren Innovationen in absehbarer Zeit von den Krankenkassen nicht mehr bezahlt werden. Dann ist es doch gut, wenn man schon einmal vorgesorgt hat und sich alles hat einbauen lassen, was vielleicht irgendwann einmal notwendig werden könnte: Aortenklappe, Schrittmacher, Hüftprothese, ICD, Augenlinse usw. Das neue Motto der Primärprävention sollte lauten: Was drin ist, ist drin, und das kann mir keiner mehr wegnehmen.

Dr. Peter Stiefelhagen

\section{Unterschätzter Risikofaktor}

\section{Stress im Job erhöht das Schlaganfallrisiko}

_ Arbeitsstress erhöht das Risiko eines ischämischen Schlaganfalls. Dafür sprechen die Ergebnisse einer Metaanalyse, in der die Daten von mehr als 138.000 Studienteilnehmern ausgewertet wurden: Der Anteil der Schlaganfälle, die sich mit einem hohen Stressniveau in der Arbeit in Verbindung bringen ließen, betrug insgesamt 4,4\% (population attributable risk, PAR). Besonders gefährdet waren Frauen: Ein stressiger Job erhöhte das Risiko um relative 33\% (PAR 6,5\%). Bei Männern war der relative Risikoanstieg mit 26\% dagegen nicht signifikant. Die Ursache für das erhöhte Schlaganfallrisiko könnte in einem stressbedingten, ungesunden Lebensstil liegen.

- Huang Y et al. Neurology ${ }^{\oplus 2015}$, online 14. Oktober; doi: 10.1212/WNL.0000000000002098

\section{Chronische Heiserkeit}

\section{Bei Laryngitis lohnt sich ein Allergietest}

_ Patienten mit chronischem Husten, Heiserkeit und häufigem Räuspern werden nicht selten erfolglos gegen Sodbrennen behandelt. Häufig leiden sie aber an einer Allergie - verursacht durch Staubmilben.

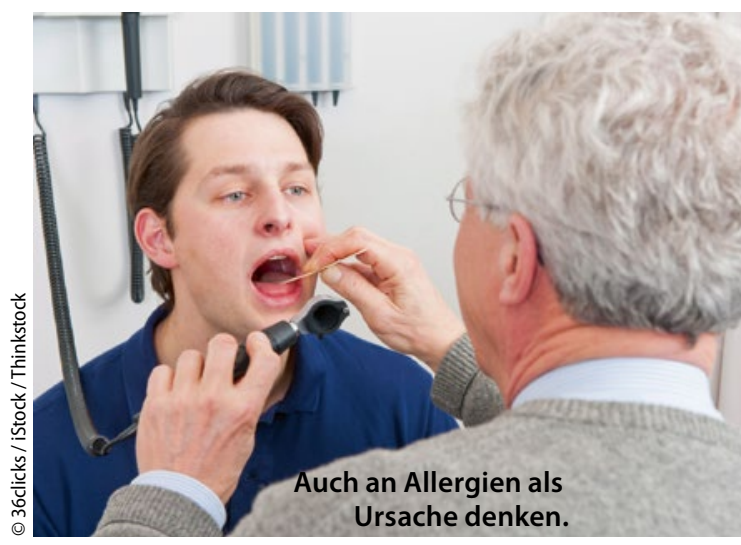

Erst wenn die Patienten auf die Refluxtherapie nicht ansprechen, würden sie vielleicht zum Allergietest geschickt. Dann wird aber ähnlich häufig eine $\mathrm{Al}$ lergie diagnostiziert wie bei Patienten mit Rhinitis oder Sinusitis, berichten Ärzte um Dr. Christopher Brook vom Boston University Medical Center. Sie werteten in einer retrospektiven Analyse die Daten von knapp 1.000 Patienten aus, die sich aufgrund diverser HNO-Beschwerden einem In-vitro-Allergietest unterzogen hatten. Mehr als die Hälfte der Betroffenen mit Laryngitis (15 von 27) waren zuvor erfolglos gegen Reflux behandelt worden war. 9 von 14 Laryngitispatienten reagierten allergisch auf Hausstaubmilben, sechs auf Gräser, fünf auf Baumpollen. Eine ähnliche Verteilung war auch bei den Rhinitis- und Sinusitispatienten zu beobachten.

mut

- Brook CD et al. Otolaryngol Head Neck Surg 2015, online 1. Oktober; doi: 10.1177/0194599815607850 